\title{
Acknowledgement to reviewers 2019
}

The Editor, Ralf Schulze, and the Publisher, the British Institute of Radiology, most gratefully acknowledge the contribution of the following colleagues who have acted unstintingly as reviewers for DMFR in 2019.

$\begin{array}{ll}\text { Ahmed Abdel Razek } & \text { Antigone Delantoni } \\ \text { Ahmad Abdelkarim } & \text { Husniye Demirturk Kocasarac } \\ \text { Murillo Abreu Jr. } & \text { Thomas Deserno } \\ \text { Hüseyin Akan } & \text { Karina Devito } \\ \text { Zuhre Akarslan } & \text { Zeng Donglin } \\ \text { Seçil Aksoy } & \text { Paulo dos Santos } \\ \text { Miguel Alcaraz } & \text { Nicholas Drage } \\ \text { Asma'a Al-Ekrish } & \text { Lars Ebert } \\ \text { Banu Alicioglu } & \text { Georg Eggers } \\ \text { Ghaida AlJamal } & \text { Annika Ekestubbe } \\ \text { Veeratrishul Allareddy } & \text { Tarek El-Bialy } \\ \text { Gonçalves Andrea } & \text { Ibrahim El-Hakim } \\ \text { Johan Aps } & \text { Brendan Fanning } \\ \text { Masao Araki } & \text { Mary Farid } \\ \text { Yoshiko Ariji } & \text { Allan Farman } \\ \text { Eiichiro Ariji } & \text { Xin Feng } \\ \text { Rieko Asaumi } & \text { Fabio Ferretti } \\ \text { Ulkem Aydin } & \text { Lennart Flygare } \\ \text { Rika Baba } & \text { Vania Fontanella } \\ \text { B.Guniz Baksi } & \text { Silvina Friedlander-Barenboim } \\ \text { Wouter Bauters } & \text { Zhipu Ge } \\ \text { Talia Becker } & \text { Arthur Goren } \\ \text { Erika Benavides } & \text { Crawford Gray } \\ \text { Erwin Berkhout } & \text { Pelin Güneri } \\ \text { Manish Bhagania } & \text { Xiaolong Guo } \\ \text { Silvio Bianchi } & \text { Anne Harwood } \\ \text { Paolo Boffano } & \text { Stefan Hassfeld } \\ \text { Richard Bohay } & \text { Yoshihiko Hayakawa } \\ \text { Michael Bornstein } & \text { Ebba Helmrot } \\ \text { Peter Bottenberg } & \text { Min-Suk Heo } \\ \text { Andres Briner } & \text { Vicente Hernandez-Soler } \\ \text { Dan Bruellmann } & \text { Charles Hildebolt } \\ \text { Jacqueline Bryant } & \text { Miki Hisatomi } \\ \text { Nikola Buric } & \text { Marinka Hol } \\ \text { Sirmahan Cakarer } & \text { Reinier Hoogeveen } \\ \text { Marcelo Cavalcanti } & \text { Keith Horner } \\ \text { Rakhi Chandak } & \text { Wang Hu } \\ \text { Jahanzeb Chaudhry } & \text { Yan Huang } \\ \text { Akhilanand Chaurasia } & \text { Kyung-Hoe Huh } \\ \text { Hui Chen } & \text { Norliza Ibrahim } \\ \text { Bong Hae Cho } & \text { Dilhan Ilguy } \\ \text { Soon-Chul Choi } & \text { Reinhilde Jacobs } \\ \text { Dan Colosi } & \text { Wolfgang Jacquet } \\ \text { Livia Corpas } & \text { Fatima Jadu } \\ \text { Claudio Costa } & \text { Apirum Janhom } \\ \text { Paulo Couto Souza } & \text { Revan Joshi } \\ \text { Zahra Dalili Kajan } & \text { Catherine Kabenge } \\ \text { Annelore De Grauwe } & \text { Byung Chul Kang } \\ \text { Mehmet Kagan Degerliyurt } & \text { Mel Kantor } \\ & \end{array}$

Freny Karjodkar

Deeba Kashtwari

Rujuta Katkar

Jerry Katz

Taisuke Kawai

Emad Khan

Vikram Khanna

Shivaji Khedkar

Jo-Eun Kim

Gyu-Tae Kim

Jin-Soo Kim

Athina Kondylidou-Sidira

Suwadee Kositbowornchai

Vandana Kumar

Tohru Kurabayashi

Hiroshi Kurita

Manuel Lagravère

Ernest Lam

Tore Larheim

Cesar Lascala

Constantino Ledesma-Montes

Chena Lee

Sam-Sun Lee

Gang Li

Christina Lindh

Alf Linney

Denggao Liu

John Ludlow

Ruo-Han Ma

David MacDonald-Jankowski

Sanjay Mallya

Colin Martin

Farah Masood

George Matsopoulos

Daniel McIlgorm

Sushma Mehkri

Daniela Melo

Dale Miles

Arthur Miller

Roberto Molteni

Paul Monsour

Shumei Murakami

Giridhar Naidu

Ruhi Nalçacı

Yuri Nejaim

Sirous Nekooei

Suk Ng

Rose Ngu

Mahdi Niknami 
Hideyoshi Nishiyama

Raphael Olszewski

Galal Omami

Evelise Ono

Kaan Orhan

Neslihan Ozveren

Sreenivasulu Pattipati

Ruben Pauwels

Jaakko Peltola

Danyel Perez

Susanne Perschbacher

Alynne Pimenta

Enrique Platin

Andrea Pontual

Maria Luiza Pontual

Elham Rajaei Behbahani

Aruna Ramesh

Flávia Ramos-Perez

Yeshwant Rawal

Madeleine Rohlin

Ingrid Rozylo-Kalinowska

Izabel Rubira-Bullen
Eduardo Sannomiya

Tsukasa Sano

Kaustubh Sansare

David Sarment

Takehito Sasaki

M.Ozgur Sayin

Dirk Schulze

Ulrich Schwancke

Werner Shintaku

Maria Silva

Steven Singer

Yigit Sirin

Güner Sonmez

Rubens Spin-Neto

Yi Sun

Zhipeng Sun

Zahra Tafakhori

Akira Taguchi

Dania Tamimi

Jefferson Tanaka

Sotirios Tetradis

Victor Tieghi Neto
Meryem Toraman Alkurt

Daniel Torres-Lagares

Ricardo Urzúa

Paul van der Stelt

Bart Vandenberghe

Christian Vanhove

Suvendra Vijayan

Hiroshi Watanabe

Ann Wenzel

Stuart White

Gail Williamson

Jie Yang

Won-Jin Yi

Gulsun Yildirim

Suk-Ja Yoon

Kazunori Yoshiura

Qiang Yu

Piero Antonio Zecca

Uwe Zeller

Dong Lin Zeng

Wenjian Zhang

Ling Zhu 